Sciences sociales et coopération en Afrique : les rendez-vous manqués. Sous la direction de Jean-Pierre JACOB, Nouveaux Cahiers de l'IUED, $\mathrm{n}^{\circ}$ 10 , Genève et Paris, IUED/Presses Universitaires de France, septembre 2000, $244 \mathrm{p}$.

Loïc Barbedette

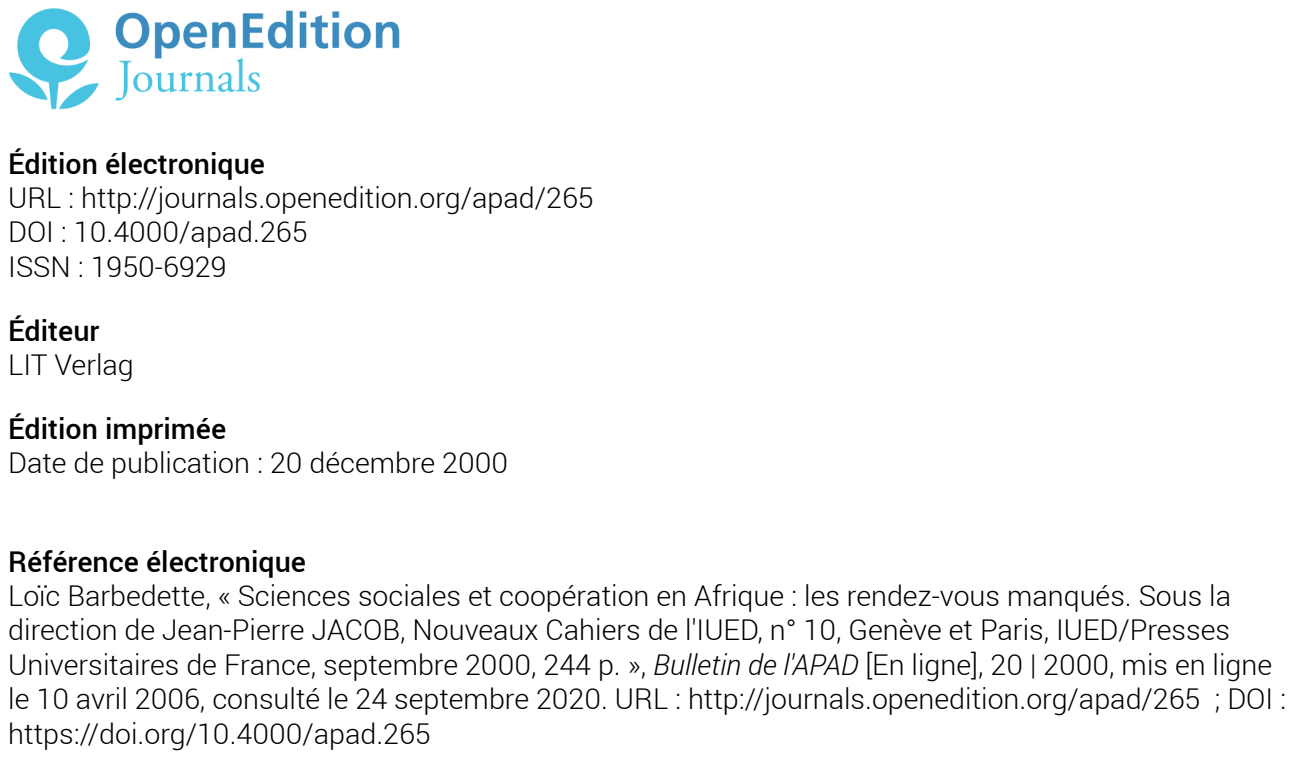

Ce document a été généré automatiquement le 24 septembre 2020.

Bulletin de I'APAD 


\title{
Sciences sociales et coopération en
} Afrique : les rendez-vous manqués. Sous la direction de Jean-Pierre JACOB, Nouveaux Cahiers de l'IUED, $n^{\circ} 10$, Genève et Paris, IUED/Presses Universitaires de France, septembre 2000, 244 p.

\author{
Loïc Barbedette
}

1 Le constat d'un rendez-vous manqué entre sciences sociales et coopération en Afrique, énoncé dans le titre de ce Cahier, peut être interprété de diverses façons, selon que l'on se situe :

- du point de vue de l'acteur inscrit dans l'univers, les pratiques et les institutions de la coopération (quel rapport le monde de la coopération, qui se suffit de connaissances non critiquées sur les sociétés où il intervient, entretient-il avec la réalité ?)

- du point de vue du chercheur produisant des connaissances sur la société (quel est le statut des sciences sociales, de la recherche et du chercheur dans les pratiques de transformation sociale en Afrique?)

- du point de vue des sociétés locales africaines (quels savoirs produisent-elles ? Dans quelles conditions? Quelle est leur fonction de contrôle dans la production et l'utilisation des connaissances par le système d'aide?)

2 Les quinze contributions réunies dans ce Cahier apportent des éclairages complémentaires utiles pour l'exposé de chacun de ces points de vue. Nous en proposerons ici une lecture de praticien confronté aux réalités du terrain et toujours questionné par elles, à la fois témoin du travail de connaissance évitée dans le monde de l'aide et du travail de connaissance contrariée chez les acteurs de la base 
(notamment dans les organisations du monde rural) aujourd'hui en Afrique. Dans une seconde partie, nous examinerons deux questions, qui nous sont suggérées par la problématique développée dans l'ouvrage. La première est celle de savoir s'il est vraiment nécessaire de s'appuyer sur une connaissance des réalités pour intervenir. Les différents constats proposés par les auteurs montrent que cette question n'est pas purement provocatrice ni fantaisiste. La seconde est de déterminer avec plus de précision qui a le plus impérativement besoin de "réussir son rendez-vous" avec la connaissance sur les réalités sociales aujourd'hui en Afrique.

Les contributions du cahierPremier constat : le savoir en sciences sociales sur les sociétés africaines existe

3 Mahaman Tidjani Alou fait l'inventaire des types de savoirs produits sur les sociétés africaines et de leurs sites de production (recherche, université, expertise en développement, mais aussi savoirs populaires et, plus récemment, savoir journalistique). Il met en évidence leur mauvaise gestion et constate qu'il n'y a "aucun lieu à partir duquel l'ensemble des constats relativement négatifs (relevés dans son article) peut être traité et des solutions élaborées pour décloisonner les différents régimes cognitifs qui se disputent le terrain en Afrique" (p.66). Relevant au passage le rapport de forces et le monopole du savoir aux mains des pays riches, il conclut sur la nécessité d'une "réflexion approfondie sur le rôle de l'expert qui peut être soit le moyen privilégié du maintien de ces inégalités. soit l'acteur clé de leur dénonciation" (p. 66).

Deuxième constat : le savoir disponible est traité avec une étonnante légèreté par la coopération au développement

Jean-David Naudet analyse le matériau informatif fourni par les statistiques provenant des sources considérées comme sérieuses des grandes agences. Il met en évidence à partir de quelques exemples bien documentés (l'appréciation de l'évolution du niveau de vie, celle de l'évolution de l'agriculture, l'évolution de la structure des économies africaines, l'ouverture de l'Afrique au marché international, l'appréciation de l'épargne) les incohérences et contradictions qui caractérisent les états de la situation qui peuvent être établis à partir de ces sources. Son propos n'est évidemment pas de critiquer le travail des statisticiens (les difficultés méthodologiques de la collecte de données statistiques dans un pays comme le Niger sont décrites par ailleurs dans un entretien de Maman Sani Sanda) mais de "rappeler que le doute sur l'information utilisée [devrait] naturellement se répercuter sur les résultats des analyses et des recommandations qui en découlent" ( $\mathrm{p}$. 53). Or, dans ce domaine, les précautions liminaires obligées des rapports d'experts sur le caractère" peu fiable" des données semblent purement incantatoires et n'empêchent nullement l'établissement de diagnostics péremptoires et l'identification de "bonnes solutions". Tout se passe finalement comme si "le manque d'information fiable était un désagrément, mais qui ne change rien au fond" (p. 31), le remède apparaissant en fin de compte comme indépendant du diagnostic.

5 Cependant, l'obstination dans l'aveuglement peut aussi conduire à des impasses dramatiques. C'est ce que démontre Peter Uvin en analysant la manière dont était construite l'image du Rwanda d'avant le génocide de 1994. A postériori, il est possible de dire que cette image occultait d'évidents indices d'accroissement de la pauvreté et ignorait les signes de montée des conflits entre Hutu et Tutsi. Il montre par ailleurs les effets de ce déni de réalité, basé sur un raisonnement circulaire (le volume d'aide consommé devient un indicateur du développement réel) et biaisé, pour la justification de l'aide externe: "ces images simplifiées. sinon totalement fausses, jouent un rôle essentiel 
dans l'opération consistant à faire d'un pays un problème de développement susceptible d'être résolu bien entendu au moyen d'une aide qui est justement celle dont disposent les institutions d'aide au développement " (p. 126).

6 L'un des objectifs de ce Cahier est de montrer comment l'"encadrement", la domination idéologique du discours développementaliste sur les connaissances, permet de s'affranchir des réalités pour imposer les solutions que l'on maîtrise ou qui arrangent (voir également, sur le sujet, Naudet, 1999). Cette pratique détourne les acteurs africains de la recherche ou de la valorisation des connaissances qui leurs seraient utiles en les assignant à "apprendre le discours de l'aide" avant d'avoir le droit de parole. Les "chercheurs" deviennent des "experts", aptes à manier une phraséologie fabriquée "ailleurs" .

7 Cet ailleurs est celui de la machinerie de la coopération internationale qui domine le marché de l'expertise et de la production des connaissances sur les sociétés "en développement". Thomas Bierschenk et Roch Mongbo, montrent à ce propos, à partir $\mathrm{du}$ cas béninois, comment, à quelques heureuses exceptions près, le travail de connaissance est assujetti aux commandes de l'aide et à la logique d'expertise. L'université elle-même doit légitimer son existence en fonction de sa contribution au "développement". Les conséquences pratiques de la monopolisation des connaissances par l'aide sont mises en évidence dans un interview de Patrick Freudiger. Il y insiste sur le fait que le tri dans les solutions qui vont ensuite se matérialiser dans les politiques (et qui est la véritable opération politique) est réalisé par les financeurs qui ont la haute main sur l'information sensible. Les acteurs africains (notamment les Etats) n'ont, pour leur part, ni la capacité d'élaborer de façon autonome des politiques, ni les moyens d'organiser un débat autour des politiques, alors qu'idéalement les priorités devraient être négociées à la fois dans leur contenu et dans leurs modalités de mise en œuvre avec tous les acteurs nationaux parties prenantes. Le développement est donc piloté de J'extérieur. En contrepoint de ces analyses, Laurent Monnier souligne, pour la République démocratique du Congo, tout l'intérêt d'une pratique de recherche empirique (celle de l'histoire immédiate) "non perturbée par les préoccupations développementalistes" (p.214), dont la tradition s'est forgée dans un cadre universitaire très tôt africanisé.

Troisième constat : les montages institutionnels et les dispositifs de développement résistent à la construction et à l'utilisation du savoir

8 L'article de Marilou Mathieu, qui analyse les rapports au savoir entretenus dans un projet de gestion des ressources naturelles au Mali, relève d'emblée ce paradoxe révélateur: dans le dispositif de planification d'un projet, les décisions financières précèdent toujours la maîtrise des informations qui seraient nécessaires pour les prendre. La planification n'est donc pas fondée sur une connaissance de la réalité, mais sur une anticipation basée sur des présupposés. Ce mauvais départ a une double conséquence.

9 D'une part, il conditionne le rapport que le projet va entretenir avec la connaissance. Tout écart avec le modèle initial devient une perturbation, et seules les informations qui ne remettent pas en question le dispositif et les modèles seront prises en compte. Le projet va produire et gérer des "méconnaissances" en organisant un système d'information visant simplement à légitimer son action. Vécus comme un danger par le projet, les savoirs sur les paysans et les savoirs paysans permettant l'interconnaissance seront ignorés (voir son analyse de l'utilisation de la MARP), filtrés ou réinventés. 
10 D'autre part, il va favoriser un repli du projet sur lui-même, un centrage des acteurs sur la défense de leurs intérêts immédiats et une exacerbation des tensions et conflits au sein du dispositif institutionnel dont la régulation va absorber l'essentiel du temps et des énergies. A ce jour, la seule réponse que les organisations internationales opposent à ce type de processus est le durcissement des procédures, qui visent à limiter l'opportunisme des acteurs de base.

Quatrième constat : les savoirs utiles ne sont pas ceux qui sont réunis dans le cadre des pratiques de développement

11 A la croisée de la production de méconnaissance et du repli sur les logiques institutionnelles, Philippe Lavigne Delville suggère que l'irresponsabilité générée par les dispositifs de développement produit de l'ignorance. A partir de l'analyse d'un projet d'aménagement hydro-agricole au sud-ouest du Burkina, conçu sans réflexion préalable sur ce qui se passera une fois les infrastructures terminées (on considère implicitement que les rendements découleront mécaniquement du potentiel de production créé par la disponibilité en eau, et que sa valorisation sera nécessairement optimale), il montre comment la machine se bloque rapidement parce que la stratégie $\mathrm{du}$ projet est mise en déroute par les tactiques paysannes. Ces dernières, qui privilégient l'équilibre de l'exploitation agricole au travers de la diversification des activités, entraînent le détournement des ressources du projet pour permettre une augmentation des rendements hors périmètre, en pluvial. Résultat non dépourvu d'intérêt, mais qui aurait pu être obtenu à moindre coût et qui ne compense pas l'impact négatif du projet décrit par ailleurs. On voit au passage combien ce projet comme tant d'actions de développement - aura été un jeu de dupes. On s'y sera d'abord joué la comédie des études préalables coûteuses et peu utiles. Toute la vie de ce projet ne sera ensuite que la poursuite d'une partie de poker menteur entre paysans faussement soumis faisant semblant de mettre en valeur le périmètre et techniciens du projet faisant semblant de réclamer le remboursement des crédits, d'un jeu du chat et de la souris entre chefs de projet, ministères successifs de tutelle, bailleurs de fonds, experts pompiers ou experts alibis.

Dans son article, P. Lavigne Delville met également en évidence la déconnexion totale entre les instances de décision et les personnes directement touchées par l'action qui débouche sur "le poids déterminant du discours, une absence sidérante de sanction par l'efficacité, et finalement une irresponsabilité généralisée quant à l'impact des activités" (p. 70), explique la reproduction de la méconnaissance dans les situations d'intervention et la difficulté à intégrer les leçons de l'expérience. La mise en lumière de cette déconnexion permet d'interroger fortement les mobiles et fonctions réelles de l'action de développement. Philippe Lavigne Delville tire pour sa part de cette analyse la conclusion que dans le cadre des projets concrets, l'apport utile des sciences sociales ne devrait pas tant porter sur la connaissance fine des sociétés locales que sur la mise à jour des jeux d'acteurs suscités par l'intervention (l'analyse des processus et des stratégies d'acteurs) et leur prise en compte dans les façons de travailler.

Cinquième constat : l'action de développement s'appuie très souvent sur des connaissances préfabriquées, construites indépendamment - voire en dépit - des résultats scientifiques

13 En appliquant la critique à la problématique de la désertification, deux contributions enrichissent l'analyse et éclairent le statut des connaissances sur lesquelles se fonde trop souvent l'action. 
Henrik Secher Marcussen montre que les connaissances scientifiques actuelles ne corroborent ni la réalité d'une avancée régulière et inéluctable du désert, ni l'existence par le passé d'un équilibre écologique aujourd'hui perturbé dans une zone toujours en tension et soumise à des changements, alors même que ces arguments sont à la base des différents discours tenus au Burkina Faso pour justifier les "stratégies de lutte contre la désertification". Il montre par ailleurs que la communauté scientifique burkinabé est trop faible pour s'affirmer face à la (mé)connaissance des experts en développement et reproduit en fait le discours consensuel dominant qui indexe la responsabilité de l'homme. Ronald Jaubert retrace pour sa part l'histoire et les avatars du concept de désertification. Il réfute lui aussi l'idée d'un équilibre antérieur perturbé par l'action de l'homme et montre, à rebours des thèses dominantes, qu'une capacité positive (et non "perturbatrice") d'adaptation des pasteurs et des agriculteurs a toujours existé. Cette dernière repose sur deux éléments : la mobilité et les liens avec les centres urbains. Or, c'est justement cette mobilité qui est contrariée depuis la période coloniale par des frontières arbitraires, cette territorialisation étant renforcée actuellement par les plans nationaux d'action contre la désertification.

Connaissance : pour qui et pour quoi?

15 Dans son introduction, Jean Pierre Jacob indique que le propos de ce Cahier est "d'explorer la validité du constat souvent opéré sur le caractère fréquemment subsidiaire, aléatoire et contingent de la mobilisation d'éléments de connaissance sur les sociétés en développement" (p. 11). Cette exploration peut prendre une allure très différente selon la définition que l'on propose des objectifs du développement. Plusieurs auteurs, qui fournissent des interprétations macro-politiques critiques de ces objectifs, concluent à l'absence de liens nécessaires entre connaissance et action.

$16 \mathrm{Si}$, en effet, comme le suggère Marilou Mathieu, les vrais intérêts des politiques de développement résident plutôt dans le contrôle militaire de certaines zones d'influence, la sujétion de régimes politiques, la limitation du déploiement d'idéologies menaçantes, la diversification des débouchés économiques pour les entreprises des pays financeurs, la maîtrise du prix des matières premières, la diminution des flux migratoires, alors "il est logique que la dissimulation de ces objectifs réels, donc la rétention de l'information, soit l'élément essentiel sur lequel achoppe toute stratégie de maîtrise de la connaissance. Les projets sont condamnés au mieux à une réussite partielle et accidentelle, au pire à un simulacre collectif, avec ou sans prise en compte du savoir sur les populations" (p. 118). Le savoir devient sans importance. La machinerie du développement n'est qu'un masque : il ne lui est pas demandé de transformer la réalité, et comme le souligne l'analyse de Jean-Pierre Jacob, "l'ignorance devient un moyen de reproduction du système" (p. 26).

17 Nul besoin non plus de prendre en compte de façon approfondie l'ensemble de la réalité sociale si le développement, selon le constat de C. Coméliau, "c'est d'abord l'expansion du système mondial productiviste, du modèle de développement qu'il diffuse et de ses conditions de réalisation que constituent l'accroissement de la consommation de marchandise, celui de investissement profitable et celui du commerce international" (p. 173). Dans ce cadre, les décideurs et les bailleurs de fonds qui "pilotent" les programmes d'aide n'ont besoin que de statistiques de base, "indicateurs de pauvreté" suffisants pour évaluer les risques qu'ils font courir aux populations, limiter la casse en finançant quelques programmes de sécurité alimentaire et de lutte contre la pauvreté et lâcher du lest quand la situation devient trop explosive. Mais Coméliau note malgré 
tout que, même de ce point de vue, les données produites pourraient être meilleures que celles qui sont produites actuellement, et que le système d'information des bailleurs n'est pas vraiment efficace, y compris du point de vue de leur propre demande.

Cependant, si par développement on entend une transformation décidée et maitrisée par les acteurs concernés, la question du rapport du développement et de ses acteurs à la connaissance se pose d'une toute autre manière : elle devient une nécessité. Ce sont alors les acteurs africains qui ont impérativement besoin non pas de recevoir, mais de développer un savoir approfondi et non dépendant sur leurs réalités.

L'article introductif de Jean-Pierre Jacob (complété en fin de Cahier par une très intéressante recension d'ouvrages sur le thème, principalement en anglais) note bien l'apparition d'un travail réflexif et critique de l'aide, produisant des études de valeur. Mais il s'agit d'un exercice à usage interne, dont l'auteur signale d'emblée toutes les limites: "on peut se demander à quoi servent en Jin de compte ces exercices d'auto-analyse lancés essentiellement par les intervenants l'ans qu'ils puissent être l'objet d'une reprise par les" bénéficiaires" (p. 27). Cette limite est en fait celle définie par la construction des rapports entre "donneurs" et "bénéficiaires". Il est nécessaire de se déplacer par rapport à elle, d'introduire une rupture pour poser en termes nouveaux la question, cette fois, du rendez-vous possible et nécessaire entre "sociétés africaines" et "sciences sociales". Ce pourrait être l'objet d'une autre livraison des Cahiers.

On peut renvoyer ici au travail théorique sur les "savoirs", les "intellectuels" et la "démocratie" en Afrique de Jean Copans (1998), qui propose lui aussi une réflexion sur la fonction de la méconnaissance dans le maintien des dépendances extérieures et des démagogies nationalistes intérieures, et sur la nécessité de l'émergence d'une véritable intelligentsia africaine. Son hypothèse est qu'il existe une série de relations entre la production (et la reproduction) de la société et l'élaboration des connaissances à propos et à destination de cette société (et il observe que s'il y a une pratique socio-intellectuelle dont on a efficacement démuni les pouvoirs africains, c'est bien la pensée sur le développement). L'inhibition d'une capacité endogène de production de savoirs et de connaissances (au-delà de leur seule "consommation" actuellement offerte par l'école et les systèmes de formation) est un puissant facteur de blocage de l'évolution de la société africaine.

On pourra également, dans cette ligne de réflexion, chercher à valoriser les tentatives discrètes, souvent étonnantes, mais socialement significatives de production de connaissances au sein de certaines organisations populaires (voir par exemple, en écho direct avec les observations de Philippe Lavigne Delville, l'élaboration d'une approche de l'exploitation familiale au sein du Réseau des organisations paysannes d'Afrique de l'Ouest - ROPPA), ou chez certains intellectuels africains "francs-tireurs".

\section{BIBLIOGRAPHIE}

Copans J. , 1998, La longue marche de la modernité africaine, Paris, Karthala. 
Naudet J.-D., 1999, Trouver des problèmes aux solutions. Vingt ans d'aide au Sahel, Paris, Editions de l'OCDE.

\section{AUTEUR}

\section{LOÏC BARBEDETTE}

Sociologue 